\title{
Barreras para acceder a los servicios de rehabilitación de la baja visión: Una revisión de tema
}

\author{
Barriers to access to low vision rehabilitation services
}

\author{
María del Pilar Oviedo'
}

Citación: Oviedo MP. Barreras para acceder a los servicios de rehabiitación de la baja visión: una revisión de tema. Ustasalud 2019;18: 48-54.

Licencia Creative Commons

\section{(c) (1) $(9)$}

\footnotetext{
${ }^{1}$ Magíster en Salud Pública, docente Facultad de Optometría, Universidad Santo Tomás, Bucaramanga, Colombia

Autor de correspondencia:

María del Pilar Oviedo

Correo electrónico:

maria.oviedo@ustabuca.edu.co
}

\section{Resumen}

Objetivo: Analizar las barreras reportadas en la literatura para el acceso a los servicios de rehabilitación de la baja visión. Materiales y métodos: Se realizó una revisión narrativa a partir de literatura científica publicada en el período 2008 a 2019 y vinculada en las bases de datos Pubmed, Scielo, Ebsco y Science Direct. Resultados: Las barreras reportadas por los prestadores de servicios de salud están relacionadas con la falta de conocimientos en baja visión y el escaso número de profesionales que se dedican a la atención, así como la invisibilizacion de la misma en los planes de atención ocular. Las barreras manifestadas por las personas están relacionadas con la falta de información y conocimiento sobre los servicios de baja visión, dificultades para el desplazamiento/transporte y el temor a los estereotipos. Conclusiones: Las barreras para acceder a los servicios de rehabilitación son complejas, y algunas pueden abordarse más fácilmente que otras. Un aspecto clave para disminuir las barreras puede ser la mejora de la formación del recurso humano y la generación de canales de comunicación entre los profesionales y las personas. El estigma asociado a la discapacidad visual es un elemento que parece ser crucial y que debe abordarse.

Palabras clave: Baja visión, rehabilitación, barreras de acceso a los servicios de salud.

\section{Abstract}

Objective: To analyze the barriers reported in the literature for access to low vision rehabilitation services. Methods: A narrative review based on scientific literature published in the period 2008 to 2019 and linked in the Pubmed, Scielo, Ebsco and Science Direct databases was conducted. Results: The barriers reported by health care providers were related to the lack of knowledge in low vision and the limited number of professionals engaged in care as well as the invisibilation of care in eye care plans. The barriers expressed by people are related to lack of information and knowledge about low vision services, difficulties in displacement/ transport and fear of stereotypes. Conclusions: Barriers to access rehabilitation services are complex, and some can be addressed more easily than others. Improving the training of human resources, and generating channels of communication between professionals and people may reduce those barriers. Stigma associated with visual impairment is an element that appears to be crucial and needs to be addressed.

Keywords: Low vision, barriers to access of health services.
Recibido para publicación: 10 de octubre de 2019 Aceptado para publicación 30 de noviembre de 2019 


\section{INTRODUCCIÓN}

La discapacidad visual es un reto de salud pública y para el desarrollo mundial, pues esta afecta las oportunidades económicas y educativas, reduce la calidad de vida y aumenta el riesgo de muerte de quienes tienen dicha condición ${ }^{1,2}$. Según el reporte mundial de discapacidad de la Organización Mundial de la Salud (OMS) el 15\% de la población global tiene una discapacidad; es decir, cerca de 1000 millones de personas, de las cuales aproximadamente 200 millones experimentan dificultades considerables en su vida diaria ${ }^{3}$. Para el año 2015, se reportó que en el mundo existen 36 millones de personas ciegas y 216 millones con baja visión ${ }^{1}$. Clínicamente, una persona tiene baja visión cuando tiene agudezas visuales entre 20/60 y 20/400, un campo visual central inferior a 20 grados en su mejor ojo, pero cuya visión residual le permite planificar o realizar una tarea determinada ${ }^{4}$. Sin embargo, se ha demostrado que la agudeza de menos de 20/40 impide la capacidad funcional y puede justificarse la remisión de rehabilitación ${ }^{5}$. Por su parte, la discapacidad visual para visión cercana se define si la AV es inferior a N6 o N8 a $40 \mathrm{~cm}$ (tipo y tamaño de letra expresado en puntos tipográficamente, letra Times New Roman) $)^{6,7}$.

Cada vez hay más pruebas de que la discapacidad visual tiene un impacto negativo en las actividades de la vida diaria específicas de la visión, como la movilidad, la participación y otros dominios de la calidad de vida ${ }^{8}$. En comparación con individuos con visión completa, aquellos con discapacidades visuales experimentarán el doble de caídas y muerte temprana, así como tres veces más depresión? .

En este contexto, existen alternativas de rehabilitación a través de los servicios de baja visión, los cuales buscan maximizar la función visual al permitir a la persona ajustarse y desarrollar habilidades compensatorias para optimizar su funcionalidad, mejorando la calidad de vida de las personas y su estado psicosocial $^{10}$. En la rehabilitación se abordan elementos clínicos, sociales, psicológicos, emocionales, las consecuencias económicas de esta condición se prescriben con ayudas específicas, tales como lupas, telescopios, filtros que les sirven para planificar y realizar alguna tarea determinada (actividades de la vida diaria en la casa o en el trabajo: cocinar, coser, leer, pagar cuentas, entre otros) contribuyendo así a su inclusión social, educativa y laboral; disminuyendo el impacto negativo que esta condición genera en la vida de este grupo poblacional ${ }^{4}$. Por tanto, la rehabilitación visual se puede definir como un proceso que se construye de acuerdo con la identificación constante de las necesidades y demandas de los individuos, que involucra su estado actual de salud, intereses, contexto y expectativas. Las acciones deben incluir la asistencia de un equipo multidisciplinario para proporcionar apoyo oportuno y completo a las personas con discapacidades ${ }^{11}$.

La rehabilitación visual, se puede ver como una forma de inversión en capital humano, ya que contribuye al desarrollo económico, social y de la salud; pese a lo anterior y a que dicho aspecto ha sido mencionado de forma reiterativa en la declaración Universal de los Derechos Humanos, en la Convención sobre los Derechos de las Personas con Discapacidad y en el Plan VISIÓN 2020 de la Organización Mundial de la Salud, en países en desarrollo solo entre el 10\% y el $15 \%$ recibe rehabilitación ${ }^{12}$. Así mismo, solo el 5\% de la población global con baja visión tiene acceso a servicios de rehabilitación, y solo una de cada 10 personas que necesitan productos de apoyo tiene acceso, en este sentido se ha reportado que 200 millones de personas con esta discapacidad visual, carecen de acceso a dispositivos para mejorar la visión ${ }^{13,14}$.

De acuerdo con lo anterior, es importante hacer una revisión que permita describir y analizar las principales barreras reportadas en la literatura para el acceso a los servicios de rehabilitación de la baja visión publicados en artículos publicados entre 2008 y 2019.

\section{MATERIALES Y MÉTODOS}

Se realizó un estudio de revisión narrativa, con el fin de describir y analizar las principales barreras reportadas en la literatura para el acceso a los servicios de rehabilitación de la baja visión. Se llevó a cabo la búsqueda en las bases de datos Pubmed, Ebsco, Science Direct y Scielo, teniendo como ventana de observación los documentos publicados en el período de 2008 a 2019. La estrategia de búsqueda utilizó los siguientes descriptores: low visión, rehabilitation, barriers, así como el operador booleano AND. Los resultados de la búsqueda fueron filtrados para descartar los artículos duplicados. Se incluyeron artículos originales o secundarios en español e inglés, así como de cualquier 
diseño, que incluían uno o más de los descriptores mencionados en las palabras claves, el título, la introducción o el resumen. Por su parte, fueron excluidos aquellos, que en el cuerpo del artículo no hacían referencia directa a barreras para el acceso a los servicios de rehabilitación visual.

\section{RESULTADOS}

Se encontraron un total de 164 artículos con los descriptores definidos, de los que se excluyeron 150 por no cumplir con los criterios propuestos para esta revisión. Finalmente, se incluyeron 14 estudios, que fueron analizados en la presente investigación (Figura 1).

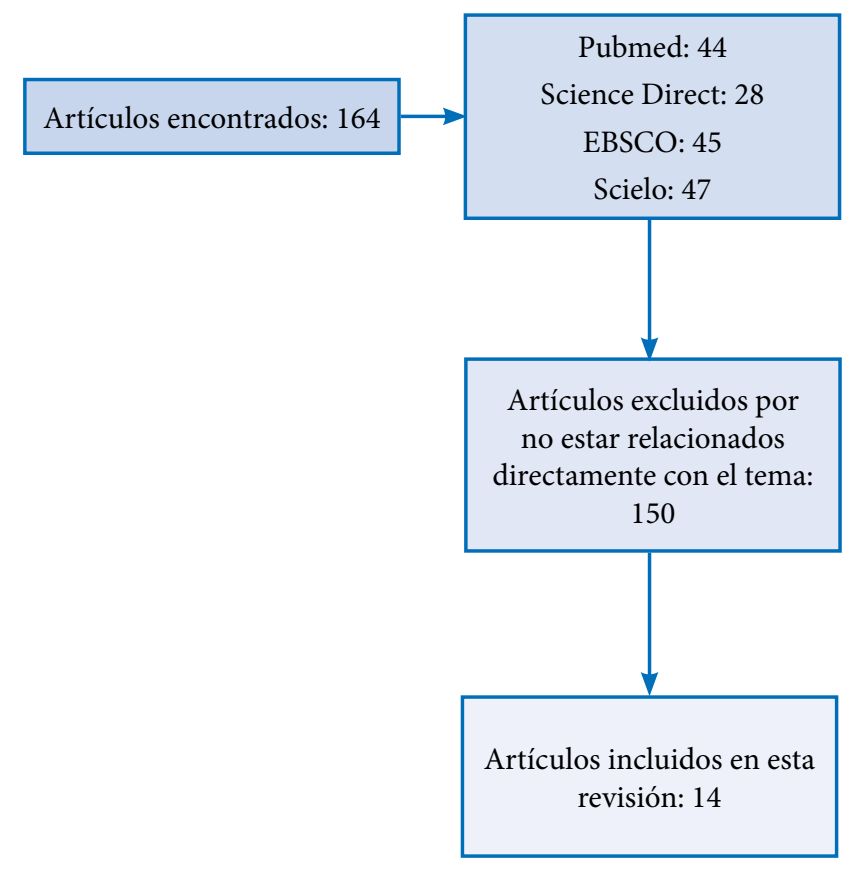

Figura 1. Diagrama de flujo de la búsqueda y selección de artículos incluidos en la revisión.

De estos, cinco artículos provenientes de Canadá, dos de Australia, dos de Estados Unidos, dos de India, uno de Colombia, uno de Nueva Guinea y uno de Sri Lanka. La Tabla 1 resume la revisión realizada de las publicaciones desde el año 2008 a 2019.

Las barreras reportadas para el acceso a los servicios de rehabilitación de la baja visión se pueden categorizar en aquellas relacionadas con las personas prestadoras de los servicios de salud (oftalmólogos, optómetras o personal de salud) y aquellas con las características relacionadas con el individuo con baja visión.
Barreras desde los prestadores de servicios de salud

De los catorce artículos seleccionados, siete incluyen la perspectiva de los prestadores de servicios. Las barreras mayormente reportadas corresponden a aquellas relacionadas con la falta de conocimientos en la temática de baja visión por parte de los profesionales y el escaso número de personas que se dedican a la atención para la rehabilitación visual, lo cual se reporta en cuatro y tres estudios, respectivamente.

Así mismo, se identifican como barreras la falta de conciencia del personal de salud, el hecho de que el cuidado para la baja visión no está incluido en los planes de salud de los países y el escaso tiempo que tienen los profesionales para la atención, lo que afecta el espacio necesario para la orientación a la persona y su familia.

\section{Barreras desde las personas con baja visión}

Nueve artículos de los incluidos en la presente revisión evidenciaron la perspectiva de las personas con baja visión. Las barreras manifestadas por las personas están relacionadas con la falta de información y conocimiento sobre los servicios de baja visión, lo cual se dio en siete estudios; otra barrera reportada corresponde a las dificultades para el desplazamiento/transporte, aspecto identificado en cinco estudios. Finalmente, el temor a los estereotipos se relaciona como otro obstaculizador para el acceso a los servicios de rehabilitación, lo cual se identifica en cuatro estudios revisados.

\section{DISCUSIÓN}

Los hallazgos permiten identificar que gran parte de los estudios sobre barreras para la rehabilitación visual han tenido lugar en países desarrollados, seis con enfoque cualitativo y ocho con enfoque cuantitativo. Se identificó el uso en un solo estudio con enfoque mixto, que pretendía dar una mirada más amplia al tema, lo cual es relevante dada la complejidad del estudio de las barreras para el acceso a servicios de salud.

Retomando los aspectos teóricos propuestos por Andersen para el estudio del acceso a los servicios de salud y específicamente de las barreras que pueden li- 


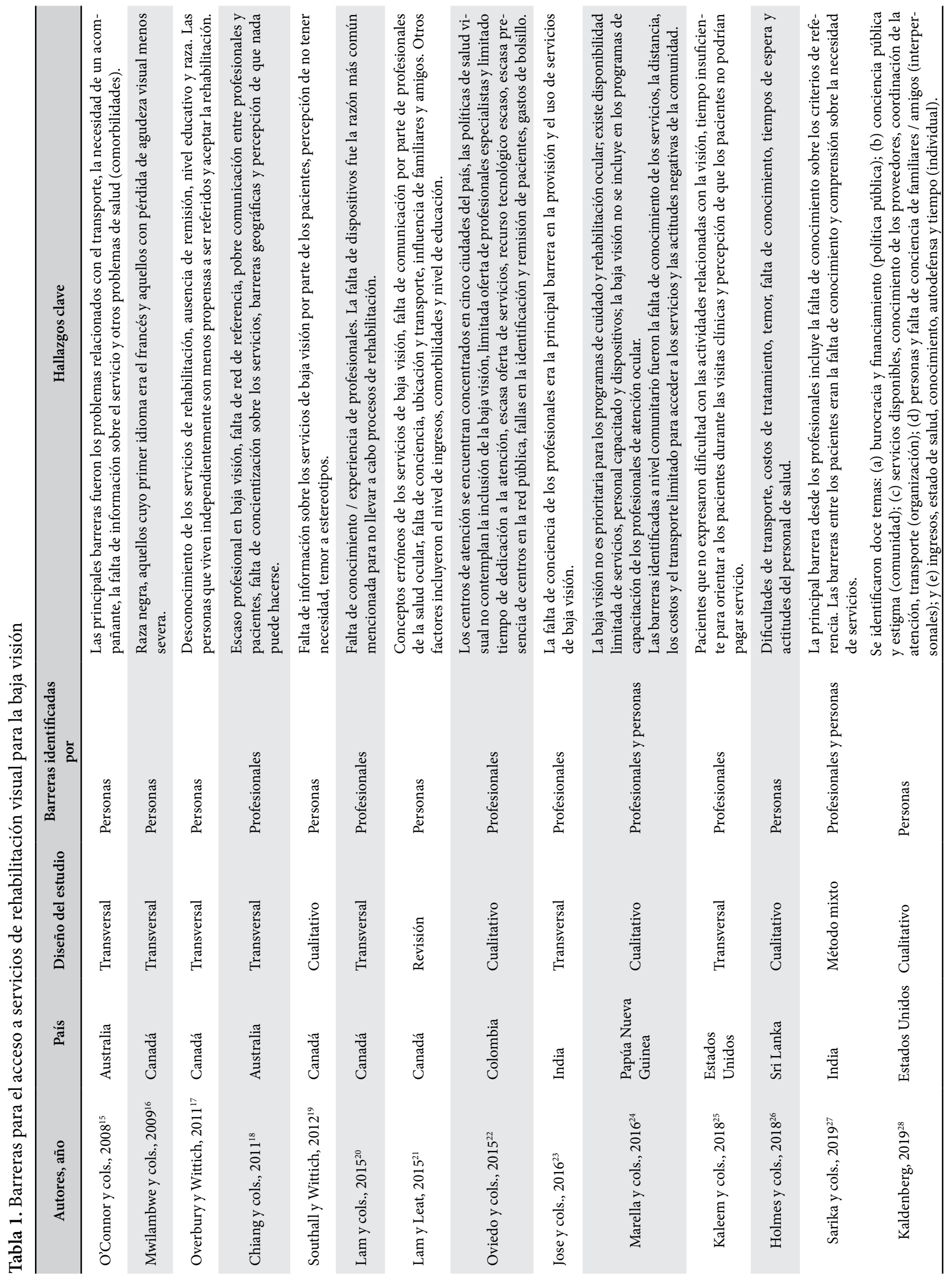


mitar el uso efectivo y eficiente, se deben explorar las miradas tanto de las personas usuarias como de los profesionales, tomadores de decisión o hacedores de política, lo cual permitirá tener un panorama amplio del fenómeno de estudio ${ }^{29}$. Pese a lo anterior, se identifica en los artículos revisados que solo dos de ellos incluyen las voces de los diferentes actores que intervienen, lo cual es un elemento que limita la comprensión de los diferentes factores que pueden entrar en juego. Por ejemplo, en el acceso intervienen múltiples factores del contexto de la política nacional, regional y local, del ámbito institucional y de los proveedores directos, lo que supone una compleja y variada red de interacciones (entre los diferentes actores institucionales de distintos niveles tomadores de decisiones, directivos de instituciones de salud y prestadores de servicios de salud), que está mediada, no solo por las realidades estructurales de los servicios (disponibilidad de recursos físicos, humanos, tecnológicos y financieros), sino por las normas y valores socialmente construidas en torno a la organización de servicios de salud que pueden condicionar el ingreso y tránsito de los usuarios en la red de atención.

Es de resaltar que una de las principales barreras reportadas en las investigaciones tiene que ver con la falta de conocimiento y entrenamiento en la temática de los profesionales de la salud, por lo cual es de vital importancia que se fortalezcan los procesos formativos del recurso humano, lo cual permitirá contar con mejores herramientas para el diagnóstico y derivación a los procesos de rehabilitación visual, los cuales son vitales para iniciar con el proceso de inclusión social. En este sentido los proveedores de atención ocular pueden ayudar a disminuir estas barreras al establecer mecanismos de comunicación con el paciente en las etapas iniciales de la pérdida de la visión con respecto a la efectividad de la rehabilitación y al hacer las derivaciones adecuadas.

Desde 1996, la Organización Mundial de la Salud ha promovido en el mundo la iniciativa VISIÓN 2020: el derecho a $v^{30}{ }^{30}$ y se cuenta con un plan de acción global para la prevención de la ceguera y la discapacidad visual evitables 2014-2019 ${ }^{31}$, que busca fomentar las políticas en salud pública y la atención integral de las personas con discapacidad visual al fortalecer la afiliación a programas de rehabilitación visual y facilitar la provisión de ayudas ópticas y no ópticas. Un aspecto critico que se identifica en los estudios revisados, tiene que ver con la invisibilización de la baja visión en el marco de las políticas de salud o los programas de atención ocular.

Por otra parte, es importante retomar las barreras reportadas por las personas con baja visión relacionadas con el temor a los estereotipos o los estigmas asociados a la discapacidad, pues de alguna u otra manera ponen en evidencia que aún subsiste la postura de la discapacidad como sinónimo de invalidez, imposibilidad, entre otros, lo cual juega un papel fundamental en el refuerzo de la minusvaloración y por lo tanto, en el mantenimiento de la discriminación. Los desafíos que enfrentan las personas con discapacidad visual son multifacéticos y complejos, los cuales a su vez reflejan normas socioculturales profundamente arraigadas que son difíciles de eliminar, como el estigma que conlleva la discapacidad ${ }^{32}$. En algunos estudios se relaciona con personas que mostraron una renuencia a etiquetarse como "ciegos" o "personas con discapacidad visual"; las personas con baja visión ven la ceguera como la ausencia total de la vista y, por eso, no sienten que deban aceptar la identidad de ser ciegos debido a su visión restante ${ }^{33}$. En este sentido, es necesario trabajar en procesos de educación, información y comunicación con enfoque de derechos y ciudadanía para contribuir en la construcción de sociedades más inclusivas.

Las barreras reportadas en los estudios pueden generar serias dificultades para el bienestar de este grupo poblacional, ya que la importancia de dar cobertura en cuanto a la rehabilitación y atención en salud redunda en la reducción de las repercusiones funcionales del déficit, la facilitación de la educación y el desarrollo de las personas con discapacidad, mantenimiento de la independencia y la actividad productiva y mejoramiento de la calidad de vida. Los servicios de salud brindados por un equipo multidisciplinario en este tipo de poblaciones son fundamentales para lograr su inclusión social, lo cual está plasmado en la Convención sobre los Derechos de las Personas con Discapacidad, en la cual se resalta la obligatoriedad de los Estados partes en proporcionar los servicios de salud que necesiten las personas con discapacidad, específicamente como consecuencia de su condición.

Esta temática cobra cada vez más importancia, pues a medida que aumenta la población adulta $\mathrm{y}$ 
adulta mayor, los problemas de visión relacionados con la edad se vuelven más comunes, por lo cual se hace más importante trabajar para el fortalecimiento de los servicios de atención en baja visión, pues se ha demostrado que el impacto en la calidad de vida de las personas que reciben rehabilitación se identifica en términos de que estos son capaces de usar su función visual, son menos dependiente de otros, y tiene menos dificultad para desarrollar actividades de la vida diaria. De la misma manera se reporta que quienes reciben estos servicios muestran una mejoría significativa en la capacidad de lectura, la movilidad visual, procesamiento de la información visual, habilidades motoras visuales, y la capacidad visual de conjunto, lo que favorece su inclusión social ${ }^{34}$.

Finalmente, se hace necesario continuar en el estudio no solo de las barreras para el acceso sino también de los facilitadores, pues estos deben ser fortalecidos, ya que se ha difundido ampliamente que la rehabilitación brinda posibilidades reales para su inclusión como miembros efectivos de la sociedad, contribuyendo así a la reducción de las desigualdades, inequidades y brechas en relación con las necesidades no satisfechas en la esfera de la salud de este grupo poblacional $^{35}$.

\section{REFERENCIAS}

1. Bourne RRA, Flaxman SR, Braithwaite T, Cicinelli MV, Das A, Jonas JB, et al. Magnitude, temporal trends, and projections of the global prevalence of blindness and distance and near vision impairment: a systematic review and meta-analysis. Lancet Glob Health. 2017;5(9):e88897. DOI: 10.1016/S2214-109X(17)30293-0.

2. Saaddine JB, Narayan KMV, Vinicor F. Vision loss: a public health problem? Ophthalmology. 2003;110(2):253-4. DOI: 10.1016/s0161-6420(02)01839-0.

3. Organización Mundial de la Salud. Informe mundial sobre la discapacidad [Internet]. WHO. [acceso: 29 de abril de 2018]. URL disponible en: http://www.who.int/ disabilities/world_report/2011/es/

4. Coco-Martin, M, Herrera-Medina, J, Cuadrado Asencio,R, De Lazaro J. Manual de Baja Visión y Rehabilitación Visual [Internet]. Editorial Médica Panamericana; 2015 [acceso: 5 de septiembre de 2018]. p. 360. URL Disponible en: https://www.medicapanamericana.com/ Libros/Libro/5417/Manual-de-Baja-Vision-y-Rehabilitacion-Visual.html
5. Pollard TL, Simpson JA, Lamoureux EL, Keeffe JE. Barriers to accessing low vision services. Ophthalmic Physiol Opt. 2003;23(4):321-7. DOI: $10.1046 /$ j.1475-1313.2003.00123.x

6. Bourne RRA, Flaxman SR, Braithwaite T, Cicinelli MV, Das A, Jonas JB, et al. Magnitude, temporal trends, and projections of the global prevalence of blindness and distance and near vision impairment: a systematic review and meta-analysis. Lancet Glob Health. 2017;5(9):e88897. DOI: 10.1016/S2214-109X(17)30293-0.

7. Fricke TR, Tahhan N, Resnikoff S, Papas E, Burnett A, Ho $\mathrm{SM}$, et al. Global prevalence of presbyopia and vision impairment from uncorrected presbyopia: systematic review, meta-analysis, and modelling. Ophthalmology. 2018; 125(10):1492-9. DOI: 10.1016/j.ophtha.2018.04.013.

8. Chiang PPC, Zheng Y, Wong TY, Lamoureux EL. Vision impairment and major causes of vision loss impacts on vision-specific functioning independent of socioeconomic factors. Ophthalmology. 2013;120(2):415-22. DOI: 10.1016/j.ophtha.2012.07.077.

9. Markowitz SN. State-of-the-art: low vision rehabilitation. Can J Ophthalmol. 2016;51(2):59-66. DOI: $10.1016 /$ j.jcjo.2015.11.002.

10. Binns AM, Bunce C, Dickinson C, Harper R, Tudor-Edwards $\mathrm{R}$, Woodhouse $\mathrm{M}$, et al. How effective is low vision service provision? A systematic review. Surv Ophthalmol. 2012;57(1):34-65. DOI: 10.1016/j.survophthal.2011.06.006.

11. da Silva MR, de Souza Nobre MIR, de Carvalho KM, Montilha R. Visual impairment, rehabilitation and International Classification of Functioning, Disability and Health. Rev Bras Oftalmol. 2014;73(5):291-301. DOI: $10.5935 / 0034-7280.20140063$.

12. World Health Organization. Rehabilitación 2030: Un llamado a la acción. Nota Conceptual. 2017;2.

13. World Health Organization. Tecnología de asistencia [Internet]. [acceso: 13 de marzo de 2019]. URL disponible en: https://www.who.int/es/news-room/fact-sheets/ detail/assistive-technology

14. World Health Organization. Lista de ayudas técnicas prioritarias [Internet]. [acceso: 13 de marzo de 2019]. URL disponible en: https://apps.who.int/iris/bitstream/ handle/10665/207697/WHO_EMP_PHI_2016.01_spa. pdf? sequence $=1$

15. O'Connor PM, Mu LC, Keeffe JE. Access and utilization of a new low-vision rehabilitation ser- 
vice. Clin Exp Ophthalmol. 2008;36(6):547-52. DOI: 10.1111/j.1442-9071.2008.01830.x.

16. Mwilambwe A, Wittich W, Freeman EE. Disparities in awareness and use of low-vision rehabilitation. Can J Ophthalmol. 2009;44(6):686-91. DOI: 10.3129/109-179.

17. Overbury $\mathrm{O}$, Wittich $\mathrm{W}$. Barriers to low vision rehabilitation: The Montreal barriers study. Invest Ophthalmol Vis Sci. 2011;52(12):8933-8. DOI: 10.1167/iovs.11-8116.

18. Chiang PP-C, O'Connor PM, Le Mesurier RT, Keeffe JE. A global survey of low vision service provision. Ophthalmic Epidemiol. 2011;18(3):109-21. DOI: 10.3109/09286586.2011.560745.

19. 19. Southall K, Wittich W. Barriers to low vision rehabilitation: A qualitative approach. J Vis Impair Blind. 2012;106(5):261-74. DOI: 10.1177/0145482X1210600502.

20. Lam N, Leat SJ, Leung A. Low-vision service provision by optometrists: a Canadian nationwide survey. Optom Vis Sci. 2015;92(3):365-74. DOI: $10.1097 /$ OPX.0000000000000512.

21. Lam N, Leat SJ. Reprint of: Barriers to accessing low-vision care: The patient's perspective. Can J Ophthalmol. 2015;50(Suppl 1):S34-9. DOI: 10.1016/j.jcjo.2015.04.007.

22. Oviedo MP, Hernández ML, Ruíz M. Baja visión en Colombia: una situación invisible para el país. Fac Nac Salud Pública. 2015;33(1):23-30.

23. Jose J, Thomas J, Bhakat P, Krithica S. Awareness, knowledge, and barriers to low vision services among eye care practitioners. Oman J Ophthalmol. 2016;9(1):37-43. DOI: 10.4103/0974-620X.176099.

24. Marella M, Yu M, Paudel P, Michael A, Ryan K, Yasmin $S$, et al. The situation of low vision services in Papua New Guinea: an exploratory study. Clin Exp Optom. 2017;100(1):54-60. DOI: 10.1111/cxo.12446.

25. Kaleem MA, West SK, Im L, Swenor BK. Referral to low vision services for glaucoma patients: Referral criteria and barriers. J Glaucoma. 2018;27(7):653-5. DOI: 10.1097/IJG.0000000000000985.

26. Holmes W, Shajehan R, Kitnasamy S, Abeywickrama C, Arsath Y, Gnanaraj F, et al. Impact of vision impairment and self-reported barriers to vision care: The views of elders in Nuwara Eliya district, Sri Lanka. Glob Public Health. 2018;13(5):642-55. DOI: $10.1080 / 17441692.2016 .1241816$.
27. Sarika G, Venugopal D, Sailaja M, Evangeline S, Krishna Kumar R. Barriers and enablers to low vision care services in a tertiary eye care hospital: A mixed method study. Indian J Ophthalmol. 2019;67(4):536-40. DOI: 10.4103/ijo.IJO_1215_18.

28. Kaldenberg J. Low vision rehabilitation services: Perceived barriers and facilitators to access for older adults with visual impairment. Br J Occupational Ther. 2019. DOI: $10.1177 / 0308022618821591$

29. Andersen RM. National health surveys and the behavioral model of health services use. Med Care. 2008;46(7):64753. DOI: 10.1097/MLR.0b013e31817a835d .

30. Promotion WHOD of CD and H, Office WHOD-GOC. State of the world's sight: VISION 2020: the right to sight: 1999-2005. 2005 [acceso: 1 de mayo de 2018]; URL disponible en: http://apps.who.int/iris/handle/10665/43300

31. World Health Organization. Salud ocular universal: un plan de acción mundial para 2014-2019 [Internet]. 2013 [acceso: 24 de febrero de 2019]. URL disponible en: https:// www.who.int/publications/list/universal_eye_health/es/

32. Tobias EI, Mukhopadhyay S. Disability and social exclusion: Experiences of individuals with visual impairments in the Oshikoto and Oshana regions of Namibia. Psychol Dev Soc. 2017;29(1):22-43. DOI: 10.1177/0971333616689203.

33. Bennion AE, Shaw RL, Gibson JM. What do we know about the experience of age-related macular degeneration? A systematic review and meta-synthesis of qualitative research. Soc Sci Med. 2012;75(6):976-85. DOI: $10.1016 /$ j.socscimed.2012.04.023.

34. McCabe P, Nason F, Demers Turco P, Friedman D, Seddon JM. Evaluating the effectiveness of a vision rehabilitation intervention using an objective and subjective measure of functional performance. Ophthalmic Epidemiol. 2000;7(4):259-70. DOI: 10.1076/opep.7.4.259.4173.

35. Cruz I. La salud colectiva y la inclusión social de las personas con discapacidad. Invest Educ Enferm. 2005;23(1):92-101. 